\title{
Position based Routing in Mobile Adhoc Networks
}

\author{
Ravi Y \\ Department of Computer Science and Engineering \\ JNTUA College of Engineering, Pulivendula \\ Y.S.R (Dist)., A.P., India.
}

\author{
M.V. Rathnamma \\ Assistant Professor \\ Department of Computer Science and Engineering \\ SSITS, Rayachoty, \\ Y.S.R. (Dist).A.P., INDIA.
}

\author{
V. Venkata Ramana \\ Associate Professor \\ Department of Computer Science and Engineering \\ SSITS, Rayachoty \\ Y.S.R. (Dist)., A.P., INDIA. \\ P. Chenna Reddy, PhD. \\ Associate Professor \\ Department of Computer Science and Engineering \\ JNTUA College of Engineering, Pulivendula \\ Y.S.R (Dist)., A.P., India.
}

\begin{abstract}
Adhoc wireless multi-hop networks (AHWMNs) are communication networks that consist of wireless nodes, placed together in an ad hoc manner, i.e. with minimal prior planning. All nodes in the network have routing capabilities and forward data packets for other nodes in multi-hop fashion. AHWMNs pose substantially different challenges to routing protocols than more traditional wired networks. AHWMN routing protocols are classified as topology-based, positionbased. Topology-based routing protocols use the information about the links that exist in the network to perform packet forwarding. Position based routing is a routing principle that relies on geographic information. Position-based routing algorithms require information about the physical position of the participating node. Commonly, each node determines its own position through the use of Global Positioning System (GPS). Decisions made based on destination position and position of forwarding nodes neighbours. A location service is used by the sender of packet to determine the position of the destination and to include it in the packet destination address.
\end{abstract}

The Greedy Perimeter Stateless Routing Protocol is a novel routing protocol for wireless datagram networks. Greedy perimeter stateless routing is based on greedy packet forwarding to forward packets to nodes that always progressively closer to the destination in each step using local information. Thus, each node forwards the message to the neighbours that are most suitable from a local point of view. Greedy forwarding can lead into a dead end, way there is no neighbour closer to the destination. The algorithm recovers by routing around the perimeter of the region by keeping state only about the local topology; GPSR scales better in perrouter state than shortest path and ad hoc routing protocols as the number of network destination increases. Under mobility's frequent topology changes, GPSR can use local topology information to find correct new routes quickly. In this paper performance of GPSR with the Ad hoc On demand distance Vector (AODV) routing protocol and Dynamic Source Routing (DSR) protocol is compared.

\section{Keywords}

Keywords - AHWMN, GPSR, Performance, AODV, DSR

\section{INTRODUCTION}

An ad hoc wireless multi-hop network (AHWMNs) is a collection of mobile devices which form a communication network with no pre-existing wiring or infrastructure. Routing in AHWMNs is challenging since there is no central coordinator that manage routing decisions. AHWMN routing protocols are classified as topology-based, position-based.

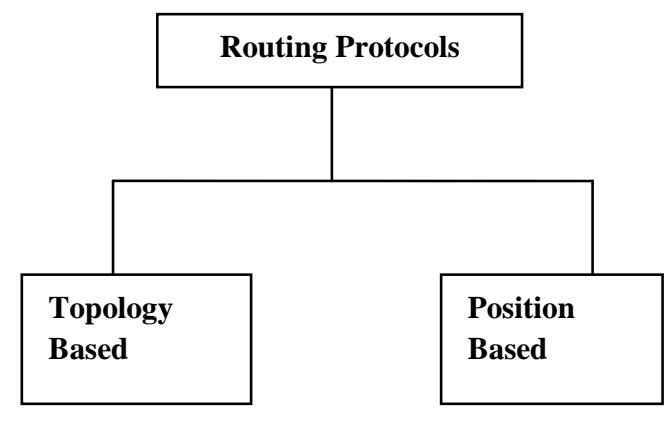

Figure1: Classification of routing protocols

Topology-based routing protocols use the information about the links that exist in the network to perform packet forwarding. They can be further divided into proactive, reactive and hybrid approaches.

Proactive algorithms employ classical routing strategies such as distance- vector routing (e.g. DSDV) or link-state routing (e.g. OLSR). They maintain routing information about the available paths in the network even if these paths are currently not used. The main drawback of these approaches is the maintenance of unusual path may occupy a significant part of the available bandwidth if the topology of the network changes frequently.

Reactive routing protocols such as AODV and DSR maintain only the routes that are currently in use and hence reduce the burden on the network. However, they still have some inherent limitations. First, since the routes are maintained only while in use, it is required to perform a route discovery before packets are exchanged between communication nodes. Second, even though route discovery is restricted to the routes currently in use, it may still generate a significant amount of 
network traffic when the topology of the network changes frequently.

Position-based routing algorithms eliminate some of the limitations of topology-based routing by using additional information. Position based routing based on idea that the source sends a message to the geographic location of destination instead of using the network address. Position based routing requires information about the physical position of participating nodes. Commonly, each node determines its own position through the use of Global Positioning System (GPS). Decisions made based on destination position and position of forwarding nodes neighbors. A location service is used by the sender of packet to determine the position of the destination and to include it in the packet destination address.

Greedy Perimeter Stateless Routing (GPSR) protocol is an efficient and scalable routing protocol in MANETs. In GPSR protocol, a node route the data packet using the locations of its one hop neighbors. When the node needs to send a data packet to destination node, it transmits the data packet to the neighbor who has the shortest distance to the destination node among all its neighbors within its transmission range. GPSR protocol uses two forwarding strategies to route the data packet to the destination. They are greedy forwarding and perimeter forwarding. GPSR makes greedy forwarding decisions using only information about a router's immediate neighbors in the network topology. When a packet reaches a region where greedy forwarding is impossible, the algorithm recovers by routing around the perimeter of the region. By keeping state only about the local topology, GPSR scales better in per-router state than shortest-path and ad-hoc routing protocols as the number of network destinations increases. Under mobility's frequent topology changes, GPSR can use local topology information to find correct new routes quickly.

In GPSR, packets are marked by their originator with their destinations' locations. As a result, a forwarding node can make a locally optimal, greedy choice in choosing a packet's next hop. Specifically, if a node knows its radio neighbors' positions, the locally optimal choice of next hop is the neighbor geographically closest to the packet's destination. Forwarding in this regime follows successively closer geographic hops, until the destination is reached.

\section{LITERATURE SURVEY}

\subsection{Dynamic Source Routing}

The Dynamic Source Routing protocol (DSR) is a simple and efficient routing protocol designed specifically for use in multi-hop wireless ad hoc networks of mobile nodes. DSR allows the network to be completely self-organizing and selfconfiguring, without the need for any existing network infrastructure or administration. Dynamic Source Routing, DSR, is a reactive routing protocol that uses source routing to send packets. It uses source routing which means that the source must know the complete hop sequence to the destination.

Each node maintains a route cache, where all routes it knows are stored. The route discovery process is initiated only if the desired route cannot be found in the route cache. To limit the number of route requests propagated, a node processes the route request message only if it has not already received the message and its address is not present in the route record of the message. DSR uses source routing, i.e. the source determines the complete sequence of hops that each packet should traverse. This requires that the sequence of hops is included in each packet's header.

\subsection{Position Based Routing Protocols}

Position based routing are

1) GFG

2) GOAFR

3) GOAFR+

4) AFR

5) OGPR

6) GPVFR

\section{7) LAR}

\subsubsection{GFG}

Nearly Stateless Routing with Guaranteed Delivery are schemes where nodes maintain only some local information to perform routing. The face routing and Greedy-Face-Greedy (GFG) schemes were described in. In order to ensure message delivery, the face routing (called perimeter algorithm) constructs a planar and connected so-called Gabriel subgraph of the unit graph, and then applies routing along the faces of the subgraph (e.g. by using the right hand rule) that intersect the line between the source and the destination. If a face is traversed using the right hand rule then a loop will be created, since a face will never exist. Forwarding in the right hand rule is performed using the directional approach.

To improve the efficiency of the algorithm in terms of routing performance, face routing can be combined with algorithms that usually find shorter routes, such as the greedy algorithm to yield GFG algorithm. Routing is mainly greedy, but if a mobile host fails to find a neighbor closer than itself to the destination, it switches the message from 'greedy' state to 'face' state.

\subsubsection{GOAFR}

A greedy routing approach is not only worth being considered due to its simplicity in both concept and implementation. Above all in dense networks such an algorithm can also be expected to end paths of good quality efficiently here, the straightforwardness of a greedy strategy contrasts highly the inexible exploration of faces inherent to face routing. For practical purposes it is inevitable to improve the performance of a face routing variant by leveraging the potential of a greedy approach. Such a combination of greedy routing and our OAFR algorithm forms Greedy Other Adaptive Face Routing GOAFR. In principle greedy routing is used as long as possible. Local minima potentially met under ways are escaped from by use of OAFR.

\subsubsection{GOAFR+}

The GOAFR+ algorithm is a combination of greedy routing and face routing. Whenever possible the algorithm tries to route greedily, that is by forwarding the message at each intermediate node to the neighbor located closest to the destination. Doing so, however, the algorithm can reach a local minimum with respect to the distance from destination that is a node um none of whose neighbors is located closer to destination than itself. In order to overcome such a local minimum, GOAFR+ applies a face routing technique, borrowing from the Face Routing algorithm. 
Face Routing proceeds towards the destination by exploring the boundaries of the faces of a planarized network graph, employing the local right hand rule (in analogy to following the right hand wall in a maze). Additionally the algorithm restricts itself to a searchable area occasionally being resized during algorithm execution.

\subsubsection{AFR}

The basis of this algorithm is formed by Face Routing. At the heart of Face Routing lies the exploration of the boundaries of faces in a planar graph, employing the local right hand rule (in analogy to following the right hand wall in a maze). On its way around a face, the algorithm keeps track of the points where it crosses the line connecting the source and the destination. Having completely surrounded a face, the algorithm returns to the one of these intersections lying closest to the destination, where it proceeds by exploring the next face closer to destination. If the source and the destination are connected, Face Routing always ends a path to the destination.

\subsubsection{OGPR}

OGPR is an efficient and scalable routing protocol, that inherits the well known techniques for routing,

\section{1) Greedy forwarding \\ 2) Reactive route discovery \\ 3) Source routing}

In this, protocol source node utilizes the geographic topology information obtained during the location request phase to establish geographic paths to their respective destinations.

\subsubsection{GPVFR}

In this section we describe Greedy PVFR, a non-oblivious routing algorithm that does not require the participating nodes to have complete face information. GPVFR is designed as a tri-modal algorithm with the following modes

- Greedy: greedy forwarding using neighbor information,

- OPVFR: greedy forwarding using face information, and

- $\quad$ Perimeter: perimeter traversal (as in GPSR).

Under GPVFR, packets are first routed in Greedy mode. When greedy forwarding to an immediate neighbor fails, a node may find that it knows of another node along its planar faces that is nearer to the destination than itself.

\subsubsection{LAR}

The Location Aided Routing proposal does not define a location-based routing protocol but instead proposes the use of position information to enhance the route discovery phase of reactive ad hoc routing approaches. Reactive ad hoc routing protocols frequently use flooding as a means of route discovery.

Under the assumption that nodes have information about other nodes' positions, this position information can be used by LAR to restrict the flooding to a certain area. This is done in a fashion similar to that of the DREAM approach. When node $\mathrm{S}$ wants to establish a route to node $\mathrm{D}, \mathrm{S}$ computes an expected zone for $\mathrm{D}$ based on available position information. If no such information is available LAR is reduced to simple flooding. If location information is available (e.g., from a route that was established earlier), a request zone is defined as the set of nodes that should forward the route discovery packet.

The request zone typically includes the expected zone. The first is a rectangular geographic region. In this case, nodes will forward the route discovery packet only if they are within that specific region. The second is defined by specifying (estimated) destination coordinates plus the distance to the destination. In this case, each forwarding node overwrites the distance field with its own current distance to the destination. A node is allowed to forward the packet again only if it is at most some $\delta$ (system parameter) farther away than the previous node.

\section{SIMULATION 3.1 Methodology}

To test and compare the performance of Greedy perimeter routing protocol, the network simulator NS-2, version 2.29 is used. The network model used in simulation is composed by mobile nodes and links that are considered wireless. Each node considered as communication end-point is host and a forwarding unit is router.

In addition to NS-2, a set of tools, mainly shell scripts and AWK filters, to post-process the output trace files generated by the simulator are developed. In order to evaluate the performance, multiple experiments have been set up.

\subsection{Metrics}

\subsubsection{Packet Delivery Ratio}

This is the ratio of total no of packets successfully received by the destination nodes to number of packets sent by the source nodes throughout the simulation.

\subsubsection{Average Routing Overhead}

This is defined as the average delay in transmission of a packet between two nodes.

\subsubsection{Average End-to-End Delay}

It is the cumulative statistical measure of the delays experienced by packets traveling between source and destination.

\subsubsection{Packet Drop}

Packet loss describes an error condition in which data packets appear to be transmitted correctly at one end of a connection, but never arrive at the other end. There might be different reasons like corrupted packets dropping the nodes, the link between nodes is not working, insufficient bandwidth, etc.

\subsection{Simulation Results}

The following results shows the Packet Delivery Ratio, Packet Drop, Average Routing Overhead and End-to-End Packet Delay of GPSR, AODV and DSR at pause times $0,10,20,50,75$ and 90 with 100 nodes. 


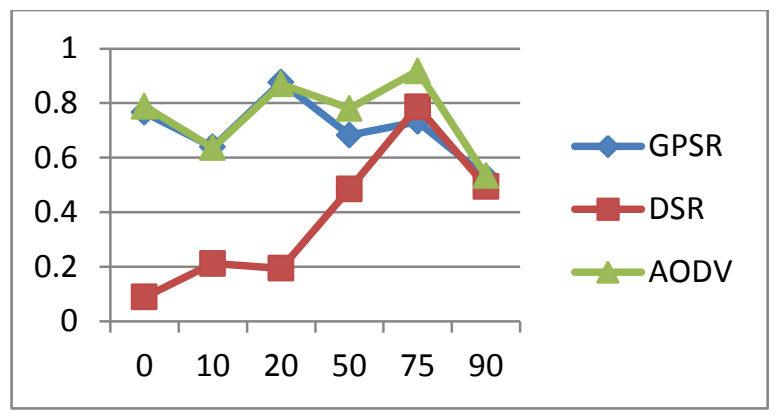

Figure 2: Packet Delivery Ratio vs Pause Times

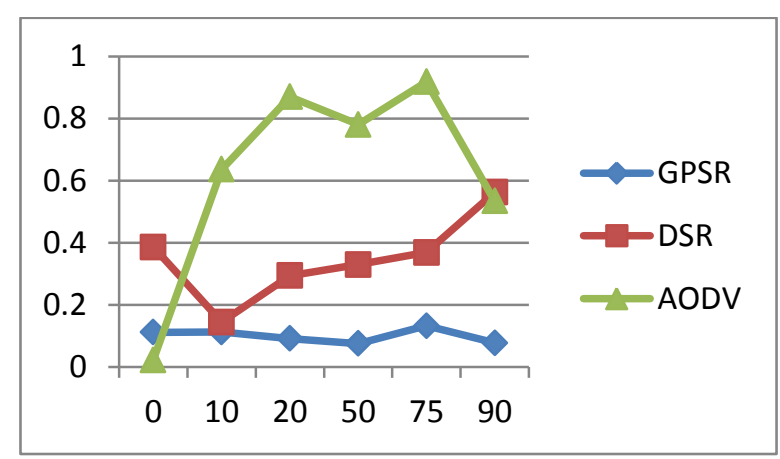

Figure 3: Packet Drop vs Pause Times

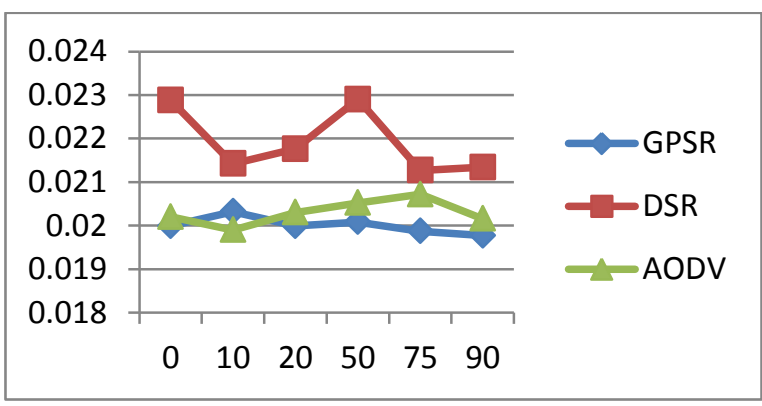

Figure 4: Average Routing Overhead vs Pause Times

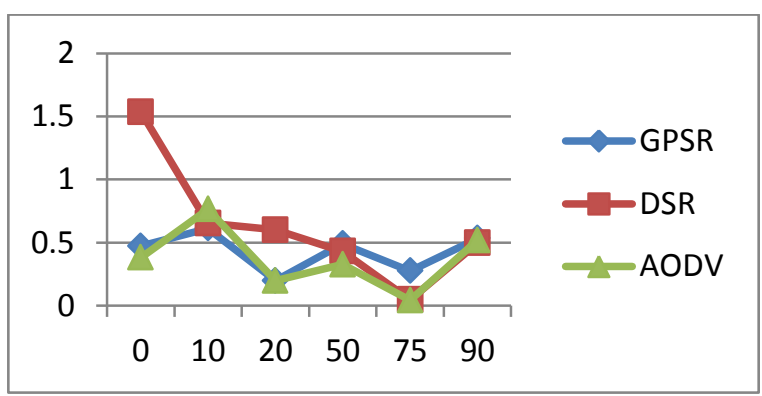

Figure 5: Average End-to-End Packet Delay vs Pause Times

The following table shows the packet delivery ratio of GPSR, AODV and DSR at different pause times 0, 10,20,50,75 and 90with 100 nodes.
Table 1. Packet Delivery Ratio at different pause times 0, $10,20,50,75$ and 90 with 100 nodes.

\begin{tabular}{|c|c|c|c|}
\hline \multicolumn{4}{|c|}{ Packet Delivery Ratio } \\
\hline & GPSR & DSR & AODV \\
\hline 0 & 0.76576 & 0.08931 & 0.78815 \\
\hline 10 & 0.63913 & 0.21174 & 0.6362 \\
\hline 20 & 0.87678 & 0.19459 & 0.8698 \\
\hline 50 & 0.68239 & 0.48586 & 0.78035 \\
\hline 75 & 0.73192 & 0.78685 & 0.91797 \\
\hline 90 & 0.52898 & 0.49475 & 0.53439 \\
\hline
\end{tabular}

Table 2. Packet Drop at different pause times $0,10,20,50,75$ and 90 with 100 nodes.

\begin{tabular}{|c|c|c|c|}
\hline \multicolumn{4}{|c|}{ Packet Drop } \\
\hline & GPSR & DSR & AODV \\
\hline 0 & 0.1117 & 0.38619 & 0.02225 \\
\hline 10 & 0.11207 & 0.14447 & 0.6362 \\
\hline 20 & 0.09148 & 0.29395 & 0.8698 \\
\hline 50 & 0.0752 & 0.32953 & 0.78035 \\
\hline 75 & 0.13248 & 0.3688 & 0.91797 \\
\hline 90 & 0.07756 & 0.56265 & 0.53439 \\
\hline
\end{tabular}

Table 3. Average Routing Overhead at different pause times $0,10,20,50,75$ and 90 with 100 nodes.

\begin{tabular}{|c|c|c|c|}
\hline \multicolumn{4}{|c|}{ Average Routing Overhead } \\
\hline & GPSR & DSR & AODV \\
\hline 0 & 0.01999 & 0.02288 & 0.0202 \\
\hline 10 & 0.02032 & 0.021423 & 0.0199 \\
\hline 20 & 0.01999 & 0.02176 & 0.0203 \\
\hline 50 & 0.02008 & 0.0229 & 0.02052 \\
\hline 75 & 0.01987 & 0.02127 & 0.02072 \\
\hline 90 & 0.01977 & 0.02134 & 0.02016 \\
\hline
\end{tabular}


Table 4. Average End-to-End Delay at different pause times $0,10,20,50,75$ and 90 with 100 nodes.

\begin{tabular}{|c|c|c|c|}
\hline \multicolumn{4}{|c|}{ Average End-to-End Delay } \\
\hline & GPSR & DSR & AODV \\
\hline 0 & 0.01999 & 0.02288 & 0.0202 \\
\hline 10 & 0.02032 & 0.021423 & 0.0199 \\
\hline 20 & 0.01999 & 0.02176 & 0.0203 \\
\hline 50 & 0.02008 & 0.0229 & 0.02052 \\
\hline 75 & 0.01987 & 0.02127 & 0.02072 \\
\hline 90 & 0.01977 & 0.02134 & 0.02016 \\
\hline & & & \\
\hline
\end{tabular}

\section{CONCLUSION}

In this paper position based routing (GPSR) and Ad hoc On demand distance Vector (AODV) routing protocol and Dynamic Source Routing (DSR) protocol are simulated over MANET using ns-2, and the performance metrics Packet delivery ratio, Average end-to-end delay, Average routing overhead, Packet drop are used. GPSR performance is comparable to that of AODV and DSR when packet delivery ratio is considered as metric. GPSR performance is better than AODV and DSR when other metrics are considered.

\section{REFERENCES}

[1] BRAD NELSON KARP "Thesis on geographic routing in wireless networks" Harvard university Cambridge, Massachusetts October 2000.

[2] MARTIN MAUVE AND JORG WIDMER, University of Mannheim Hannes Hartenstein, NEC Europe, Heidelberg, "A Survey on Position-Based Routing in Mobile Ad Hoc Networks", IEEE Network, November/December 2001.

[3] YOUNG-BAE KO, NITIN H. VAIDYA "LocationAided Routing (LAR) in mobile ad hoc networks", J.C. Baltzer AG, Science Publishers, USA.

[4] MARTIN MAUVE AND JORG WIDMER, university of Mannheim Hannes Hartenstein, NEC Europe, Heidelberg, "A Survey on Position-Based Routing in Mobile Ad Hoc Networks", IEEE Network, November/December 2001.

[5] LI J, JANNOTI J, DECOUTO D, KARGER D, AND MORRIS R, "A Scalable Location Service for geographic ad-hoc routing", Conference On Mobile Computing and Networking (MobiCom 2000), Boston, MA, USA, August 2000. 